\title{
Effect of long non-coding RNA AK021443 on promoting hepatic fibrosis in vitro
}

\author{
YUXIN YANG $^{1}$ and ZONGSU MIN ${ }^{2}$ \\ ${ }^{1}$ Clinical Laboratory of Zunyi Maternity and Child Health Care Hospital; ${ }^{2}$ Department of Blood Transfusion, \\ Zunyi Maternity and Child Health Care Hospital, Zunyi, Guizhou 563000, P.R. China
}

Received April 15, 2020; Accepted August 10, 2020

DOI: $10.3892 / \mathrm{mmr} .2021 .11835$

\begin{abstract}
Long non-coding (lnc)RNAs serves an important role in the occurrence and development of hepatic fibrosis. lncRNA AK021443 is highly expressed in hepatocellular carcinoma (HCC) and promotes HCC cell proliferation, invasion and migration. The present study aimed to investigate the effect of AK021443 on hepatic fibrosis. AK021443 was overexpressed in the human LX-2 hepatic stellate cell (HSC) line using a plasmid to observe its effect on hepatic fibrosis in vitro. A Cell Counting Kit-8 assay was performed to assess cell proliferation, whereas cell cycle distribution and related proteins were analyzed via flow cytometry and western blotting, respectively. The protein expression levels of epithelial-mesenchymal transition (EMT)-associated and extracellular matrix (ECM) proteins were also analyzed via western blotting. Immunofluorescence was conducted to observe the generation of collagen 1 , and the activity of inflammatory factors and reactive oxygen species (ROS) was also analyzed. Compared with the pcDNA group, AK021443 overexpression significantly promoted cell proliferation, enhanced the transition of cells from $G_{1}$ to $S$ phase and increased the expression of cyclin-dependent kinase 2 and cyclin D1, but reduced the p21 protein expression levels. In addition, EMT capabilities, ECM deposition and the generation of collagen1 were increased by AK021443 overexpression compared with the pcDNA group. Moreover, AK021443 overexpression significantly increased the release of inflammatory cytokines, including TGF- $\beta$, interleukin- $\beta$, platelet derived growth factor, epidermal growth factor and ROS, compared with the pcDNA group. In conclusion, the present study suggested that AK021443 overexpression increased HSC proliferation, activation and the proinflammatory response, indicating the potential role of AK02144 in aggravating hepatic fibrosis.
\end{abstract}

Correspondence to: Dr Yuxin Yang, Clinical Laboratory of Zunyi Maternity and Child Health Care Hospital, 287 North Zhonghua Road, Zunyi, Guizhou 563000, P.R. China E-mail: yyuxin20@163.com

Key words: long non-coding RNA, LX-2, hepatic fibrosis, cell cycle, epithelial-mesenchymal transition, extracellular matrix

\section{Introduction}

Hepatic fibrosis is a pathological change caused by various endogenous and exogenous damaging factors, such as inflammation, bacteria, viral infections, alcohol toxicity, drug toxicity and genetic factors, resulting in chronic liver damage (1). Hepatic fibrosis manifests in the abnormal proliferation of connective tissue in the liver, excessive deposition of extracellular matrix (ECM) and the activation of hepatic stellate cells (HSCs), which is an inevitable stage for numerous chronic liver diseases, including viral hepatitis, alcoholic fatty liver and cholestatic liver disease progressing to cirrhosis (2). The activation of HSCs serves a crucial role in the progression of hepatic fibrosis (3). Under normal physiological conditions, HSCs remain stationary, with low proliferative activity and a reduced ability to synthesize collagen; however, HSCs can be activated and converted into myofibroblasts under the stimuli of damaging factors (4). Myofibroblasts can secrete fibroblast-promoting proteins, such as TGF- $\beta$, connective tissue growth factor and tissue inhibitors of metalloproteinases (TIMP), resulting in the generation of ECM, such as collagen, fibronectin and laminin, thus serving a key role in the occurrence of hepatic fibrosis (5). In China, 300 million individuals suffer from viral hepatitis (primarily caused by hepatitis B virus), non-alcoholic fatty liver disease and alcoholic hepatitis (6). Delayed treatment of hepatic fibrosis can lead to the progression of various liver diseases to cirrhosis, thus increasing the risk of concurrent acute/chronic liver failure and HCC (7). Early treatment with antifibrosis drugs can effectively reverse the process and has become a research hotspot in hepatic diseases (8).

In recent years, a large number of studies have demonstrated that long non-coding (lnc)RNAs are involved in the occurrence and development of hepatic fibrosis by regulating fibrosis-related cell signaling pathways and activating HSCs (9-11). IncRNAs are a type of RNA that are $>200$ nucleotides in length and lack protein coding abilities (10). At first, lncRNAs were considered to be the 'noise' of genome transcription with no biological function. However, increasing research indicated that lncRNAs are involved in numerous physiological and pathological processes, including cell differentiation, development, tumorigenesis, migration and organ and tissue fibrosis $(12,13)$. IncRNA AK021443 is a newly identified lncRNA, and the expression levels were found to be increased 
in tissues of patients with HCC (14), whereas AK021443 knockdown inhibits HCC cell proliferation, invasion and migration (15). However, whether AK021443 participates in the regulation of hepatic fibrosis is not completely understood.

lncRNA research has primarily focused on tumors; however, increasing research has been conducted to investigate the role of lncRNAs in fibrotic diseases $(16,17)$. A recent study suggested that lncRNAs are specifically expressed in a variety of fibrotic tissues and serve an important regulatory role in the occurrence and development of fibrosis (9). Therefore, identifying the regulatory mechanism underlying lncRNAs in fibrotic diseases and their potential value in the prevention and treatment of fibrosis is of significance for the clinical treatment of fibrotic diseases.

The aim of the present study was to investigate the effects of AK021443 overexpression on HSC proliferation and activation in the LX-2 cell line.

\section{Materials and methods}

Cell culture. The human LX-2 HSC cell line (American Type Culture Collection) was cultured in RPMI-1640 (Gibco; Thermo Fisher Scientific, Inc.) supplemented with 10\% FBS (Wisent, Inc.) and $1 \%$ streptomycin/penicillin antibiotics at $37^{\circ} \mathrm{C}$ with $5 \% \mathrm{CO}_{2}$. The medium was replaced every other day. At $80-90 \%$ confluence, cells were passaged and cells in the logarithmic growth phase were used for subsequent experiments.

Plasmid construction and transfection. Recombinant full-length human AK021443 cDNA that was cloned into the pcDNA3.1 vector (pcDNA-AK021443) was designed and synthesized by Shanghai GenePharma Co.,Ltd. The pcDNA3.1 empty vector was used as a negative control (Shanghai GenePharma Co., Ltd.). Cells at the density of $2 \times 10^{6} / \mathrm{ml}$ were transfected with $2 \mu \mathrm{g} / \mathrm{ml}$ plasmids using Lipofectamine ${ }^{\circledR} 2000$ (Invitrogen; Thermo Fisher Scientific, Inc.) according to the manufacturer's instructions. At $72 \mathrm{~h}$ post-transfection, cells were used for subsequent experiments.

Cell proliferation assay. To determine cell proliferation, cells were cultured in 96-well plates and transfected with indicated vectors. At $0,24,48$ and $72 \mathrm{~h}$ post-transfection, cell proliferation was assessed using the Cell Counting Kit-8 (CCK-8) assay (Beyotime Institute of Biotechnology) according to the manufacturer's instructions. Briefly, $10 \mu \mathrm{l} \mathrm{CCK-8} \mathrm{working}$ solution was added to each well and incubated for $2 \mathrm{~h}$ at $37^{\circ} \mathrm{C}$. Absorbance was measured at a wavelength of $450 \mathrm{~nm}$ using a microplate reader.

Cell cycle analysis. Flow cytometry was performed to analyze the effect of AK021443 on the cell cycle. Briefly, LX-2 cells were fixed with $70 \%$ ethanol overnight at $4{ }^{\circ} \mathrm{C}$ and incubated with $0.5 \mathrm{mg} / \mathrm{ml}$ RNaseA (Thermo Fisher Scientific, Inc.) at $37^{\circ} \mathrm{C}$ for $30 \mathrm{~min}$. Following incubation with $25 \mu \mathrm{g} / \mathrm{ml}$ propidium iodide (Thermo Fisher Scientific, Inc.) on ice for $1 \mathrm{~h}$ in the dark, the cell cycle distribution was analyzed using a FACSCalibur flow cytometer (BD Biosciences). Data were analyzed using a flow cytometry software (iSort Automated Cell Sorter A.0; Thermo Fisher Scientific, Inc).
Reverse transcription-quantitative PCR (RT-qPCR). Total RNA was extracted from LX-2 cells using TRIzol ${ }^{\circledR}$ reagent (Invitrogen; Thermo Fisher Scientific, Inc.). Total RNA $(1 \mu \mathrm{g})$ was reverse transcribed into cDNA $\left(37^{\circ} \mathrm{C}\right.$ for $15 \mathrm{~min}$ and $85^{\circ} \mathrm{C}$ for $5 \mathrm{sec}$ ) using the PrimeScript RT reagent kit with gDNA Eraser (Takara Biotechnology Co., Ltd.). Subsequently, qPCR was performed using the TB Green Fast qPCR Mix (Takara Biotechnology Co., Ltd.). The following primers were used for qPCR: AK021443 forward, 5'-CTTGAACCCAGAAGACAG G-3' and reverse, 5'-ATGGAACATTAGAGGTAGCAC-3'; and $\beta$-actin forward, 5'-ATCGTGCGTGACATTAAGGAGAAG-3' and reverse, 5'-AGGAAGGAAGGCTGGAAGAGTG-3'. The following thermocycling conditions were used for qPCR: Initial denaturation at $95^{\circ} \mathrm{C}$ for $2 \mathrm{~min}$; followed by 40 cycles at $95^{\circ} \mathrm{C}$ for $20 \mathrm{sec}, 58^{\circ} \mathrm{C}$ for $20 \mathrm{sec}$ and $72^{\circ} \mathrm{C}$ for $20 \mathrm{sec}$. mRNA expression levels were quantified using the $2^{-\Delta \Delta \mathrm{Cq}}$ method (18) and normalized to the internal reference gene $\beta$-actin.

Western blotting. Total protein was extracted from LX-2 cells using RIPA buffer (Beyotime Institute of Biotechnology) and quantified using the bicinchoninic acid protein assay kit (Thermo Fisher Scientific, Inc.). Equal amounts of protein $(50 \mu \mathrm{g})$ were separated via $8-12 \%$ SDS-PAGE and transferred onto PVDF membranes (Bio-Rad Laboratories, Inc.). The membranes were blocked with TBS- $0.05 \%$ Tween-20 containing $5 \%$ skimmed milk at room temperature for $2 \mathrm{~h}$. Subsequently, the membranes were incubated overnight at $4^{\circ} \mathrm{C}$ with primary antibodies targeted against: cyclin D1 (cat. no. ab16663; 1:200; Abcam), cyclin-dependent kinase 2 (CDK2; cat. no. ab32147; 1:1,000; Abcam), p21 (cat. no. ab109520; 1:5,000; Abcam), E-cadherin (cat. no. ab40772; 1:10,000; Abcam), $\mathrm{N}$-cadherin (cat. no. ab76011; 1:10,000; Abcam), vimentin (cat. no. ab92547; 1:5,000; Abcam), snail (cat. no. ab216347; 1:1,000; Abcam), TIMP1 (cat. no. ab211926; 1:1,000; Abcam), collagen1 (cat. no. ab34710; 1:10,000; Abcam), endothelin 1 (ET-1; cat. no. ab113697; 1:250; Abcam), matrix metallopeptidase (MMP)2 (cat. no. ab92536; 1:5,000; Abcam), MMP9 (cat. no. ab38898; 1:1,000; Abcam) and GAPDH (cat. no. sc-32233; 1:10,000; Santa Cruz Biotechnology, Inc.). Following primary incubation, the membranes were incubated with a goat anti-rabbit IgG horseradish peroxidase-conjugated secondary antibody (cat. no. ab205718; 1:10,000; Abcam) at room temperature for $2 \mathrm{~h}$. Protein bands were visualized using an enhanced chemiluminescence kit (Cytiva) and ImageJ software (v1.4; National Institutes of Health) was utilized to analyze the intensity of each protein band. GAPDH was used as the loading control.

Immunofluorescence (IF). LX-2 cells cultured on slides were fixed with $4 \%$ paraformaldehyde for $20 \mathrm{~min}$ at room temperature (RT), blocked with $5 \%$ bovine serum albumin (Beyotime Institute of Biotechnology) at RT for $30 \mathrm{~min}$ and incubated with an anti-collagen1 primary antibody (cat. no. ab34710; 1:200; Abcam) overnight at $4^{\circ} \mathrm{C}$. After washing with PBS, cells were incubated with a FITC-conjugated goat anti-rabbit secondary antibody (cat. no. ab6717; 1:5,000; Abcam) in the dark at $37^{\circ} \mathrm{C}$ for $1.5 \mathrm{~h}$. After washing with PBS, the slides were incubated with DAPI (RT, $5 \mathrm{~min}$ ) for nuclear staining. Stained slides were observed using a confocal fluorescent microscope (magnification, $\mathrm{x} 400$ ). 


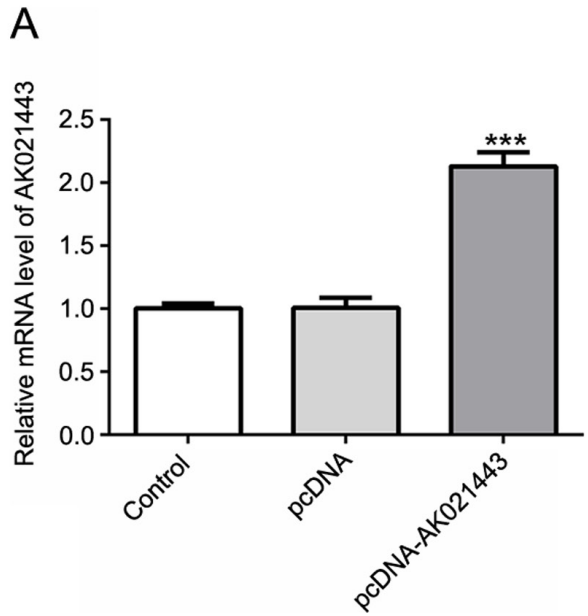

B

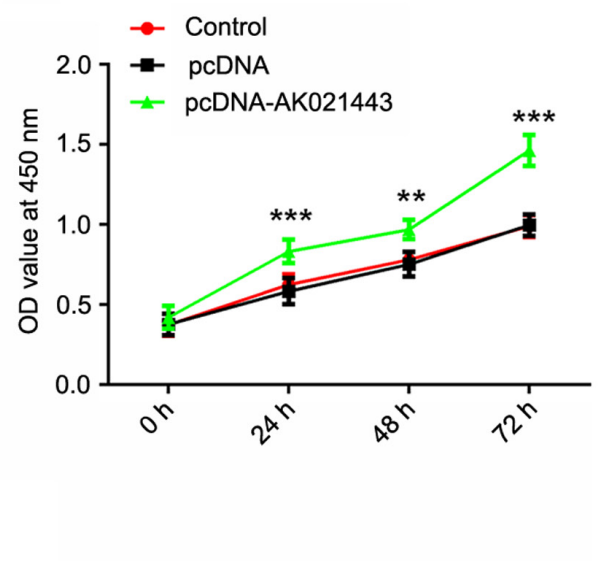

Figure 1. AK021443 overexpression increases LX-2 cell proliferation. (A) AK021443 mRNA expression levels in the different experimental groups (n=3). (B) Cell proliferation was detected at $0,24,48$ and $72 \mathrm{~h}$ post-transfection using the Cell Counting Kit- 8 assay ( $\mathrm{n}=3$ ). ${ }^{* *} \mathrm{P}<0.01$ and ${ }^{* * * *} \mathrm{P}<0.001 \mathrm{vs}$. pcDNA. OD, optical density.

Detection of inflammatory factors and oxidative stress level. The levels of the proinflammatory cytokines, TGF- $\beta$ (cat. no. ab100647), interleukin (IL)-1 $\beta$ (cat. no. ab100562), platelet derived growth factor (PDGF; cat. no. ab184860) and epidermal growth factor (EGF; cat. no. ab217772) were detected using ELISA kits from Abcam according to the manufacturer's protocol. Briefly, standard samples and cell supernatants were added into wells with corresponding antibody coating and incubated for $2 \mathrm{~h}$ at room temperature. Subsequently, conjugate was added to the wells and incubated for $2 \mathrm{~h}$ at room temperature, followed by $50 \mu \mathrm{l}$ stop solution to terminate the reaction. Absorbance was measured at a wavelength of $450 \mathrm{~nm}$ using a SpectraMax 340 microplate reader (Molecular Devices LLC). A cellular reactive oxygen species (ROS) assay kit (cat. no. ab186027; Abcam) was used to determine ROS levels. Briefly, transfected cells were plated $\left(4 \times 10^{4} / 100 \mu\right.$ l per well) in a 96 -well plate overnight. Subsequently, cells were stained with ROS red stock solution for $30 \mathrm{~min}$ at room temperature. Absorbance was measured using a microplate reader and ROS levels were determined by the fluorescence increase at $\mathrm{Ex} / \mathrm{Em}=520 / 605 \mathrm{~nm}(19)$.

Statistical analysis. All experiments were repeated at least three times and statistical analyses were performed using GraphPrism software (version 5.0; GraphPad Prism, Inc.). Data are presented as the mean $\pm \mathrm{SD}$. Comparisons among groups were analyzed using one-way ANOVA followed by Tukey's post hoc test. $\mathrm{P}<0.05$ was considered to indicate a statistically significant difference.

\section{Results}

AK021443 overexpression promotes $L X-2$ proliferation. To investigate the potential role of lncRNA AK021443 in hepatic fibrosis in vitro, AK021443 was overexpressed in the LX-2 HSC cell line to observe alterations to cellular molecular processes associated with hepatic fibrosis. The results verified the successful overexpression of AK021443 in cells transfected with pcDNA-AK021443 compared with cells transfected with pcDNA (Fig. 1A).

Cell proliferation was assessed using a CCK-8 assay, flow cytometry and western blotting. At $0,24,48$ and $72 \mathrm{~h}$ post-transfection, cell viability was assessed by performing a CCK-8 assay. The results suggested that AK021443 overexpression significantly increased cell proliferation from $24 \mathrm{~h}$ in a time-dependent manner compared with the pcDNA group (Fig. 1B). Flow cytometry was conducted to detect the cell cycle distribution, and the results indicated that pcDNA-AK021443 significantly decreased the proportion of $\mathrm{G}_{1}$ cells, but significantly increased the proportion of $\mathrm{S}$ and $\mathrm{G}_{2}$ phase cells compared with the pcDNA group (Fig. 2A). Moreover, the protein expression levels of cyclin-dependent kinase 2 (CDK2) and cyclin D1 were significantly increased, whereas p21 expression levels were significantly decreased by pcDNA-AK021443 compared with pcDNA (Fig. 2B). Therefore, the results indicated that AK021443 could increase LX-2 cell proliferation.

AK021443 overexpression triggers the activation and conversion of $L X-2$ cells into myofibroblasts. Subsequently, to investigate whether AK021443 could induce the activation of LX-2 cells and their conversion into myofibroblasts, the expression levels of proteins associated with epithelial-mesenchymal transition (EMT) and ECM were detected. The protein expression level of E-cadherin was significantly decreased, whereas $\mathrm{N}$-cadherin, vimentin and snail protein expression levels were significantly increased in AK021443-overexpression LX-2 cells compared with pcDNA-transfected cells (Fig. 3). AK021443 overexpression also significantly increased the protein expression levels of TIMP1, collagen1 and ET-1, but slightly reduced the expression levels of MMP2 and MMP9 compared with the pcDNA group (Fig. 4). In addition, IF staining was performed to assess collagen1 expression in LX-2 cells transfected with pcDNA-AK021443. The results suggested that pcDNA-AK021443 increased the levels of collagen1 compared with pcDNA, which was consistent with the western blotting results (Fig. 5). The results demonstrated 
A
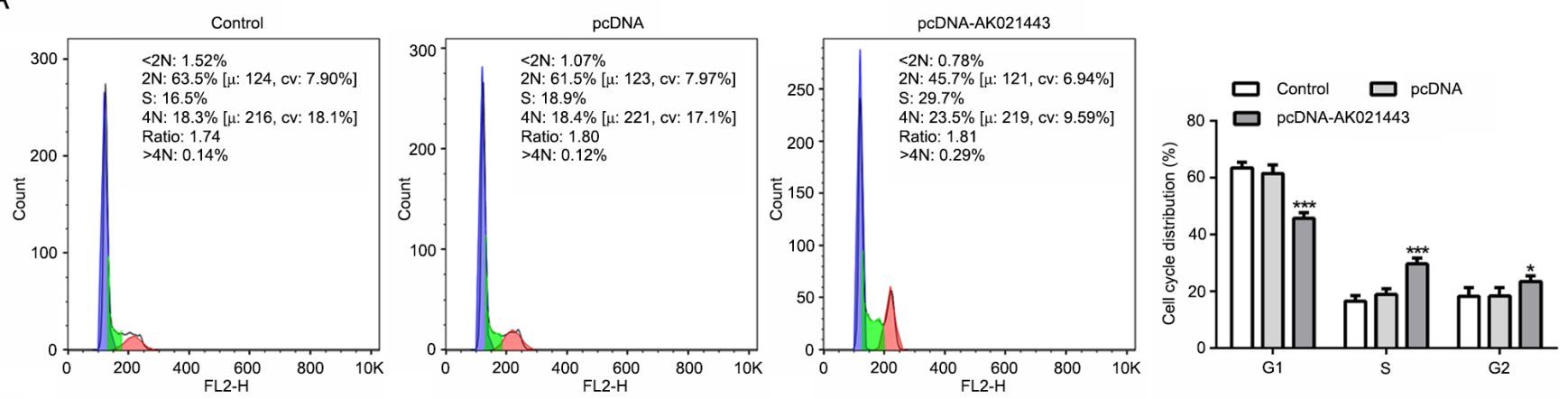

B
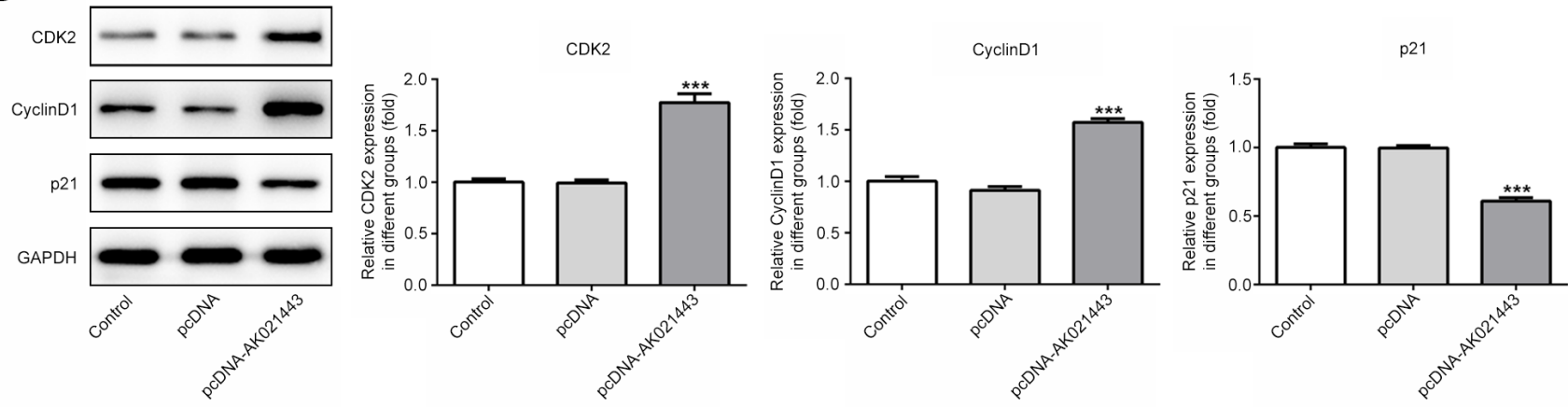

Figure 2. AK021443 overexpression facilitates LX-2 cell proliferation. (A) Cell cycle distribution was assessed via flow cytometry (n=3). (B) Expression levels of cell cycle-associated proteins were measured via western blotting $(n=3) .{ }^{*} \mathrm{P}<0.05,{ }^{* * * *} \mathrm{P}<0.001$ vs. pcDNA. CDK2, cyclin-dependent kinase 2.

A

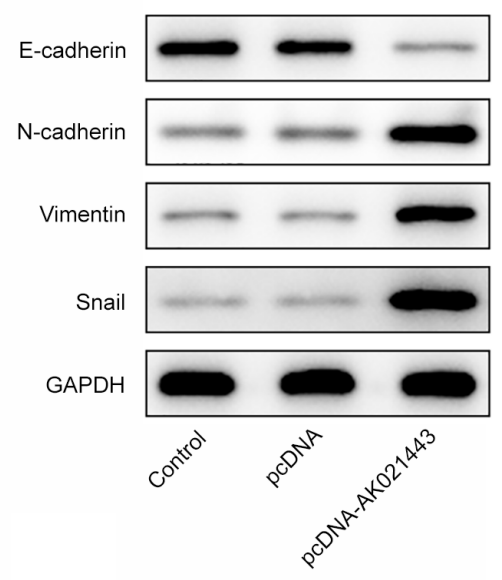

B

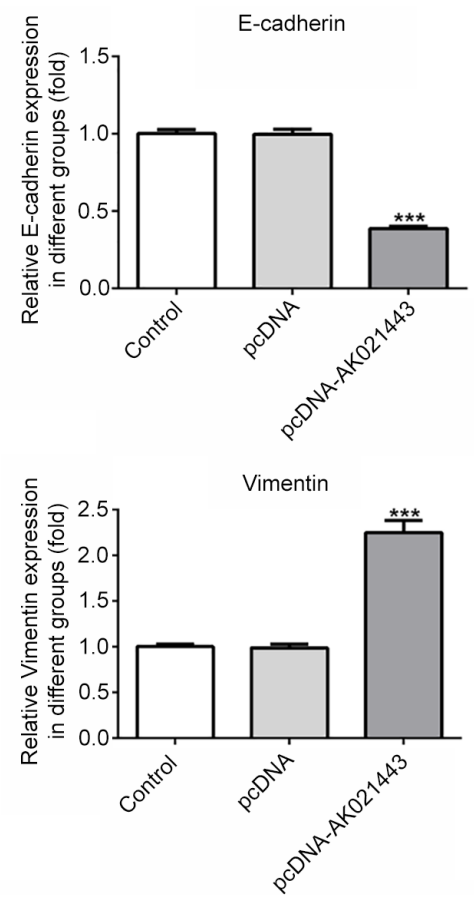

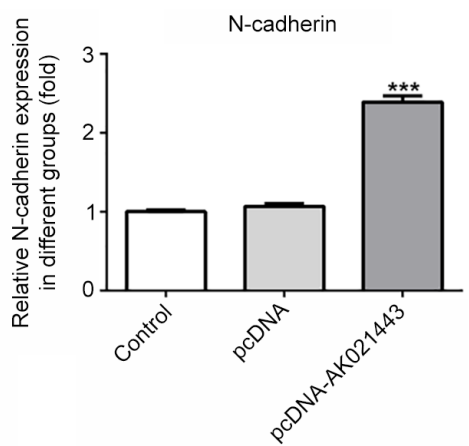

Snail

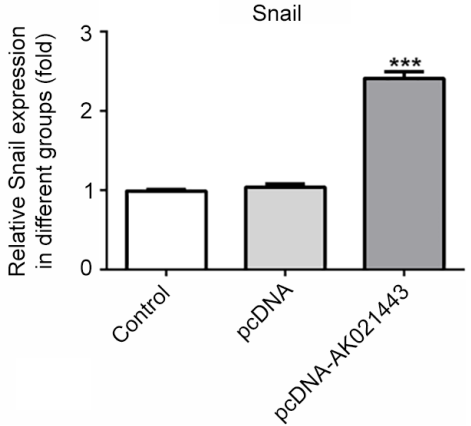

Figure 3. AK021443 overexpression increases epithelial-mesenchymal transition. Protein expression levels were (A) determined by western blotting and (B) semi-quantified for E-cadherin, $\mathrm{N}$-cadherin, vimentin and snail $(\mathrm{n}=3) .{ }^{* * *} \mathrm{P}<0.001$ vs. pcDNA.

that AK021443 may function to trigger EMT and ECM deposition in HSCs.

AK021443 overexpression enhances the generation of the inflammatory cytokines in $L X-2$ cells. Alterations to the inflammatory response in LX-2 cells were investigated.
The results indicated that the generation of IL- $1 \beta$, TGF- $\beta$, PDGF, EGF and ROS was increased by AK021443 overexpression compared with the pcDNA group (Fig. 6). The results suggested that AK021443 might display a stimulatory effect on the release of inflammatory cytokines in LX-2 cells. 
A

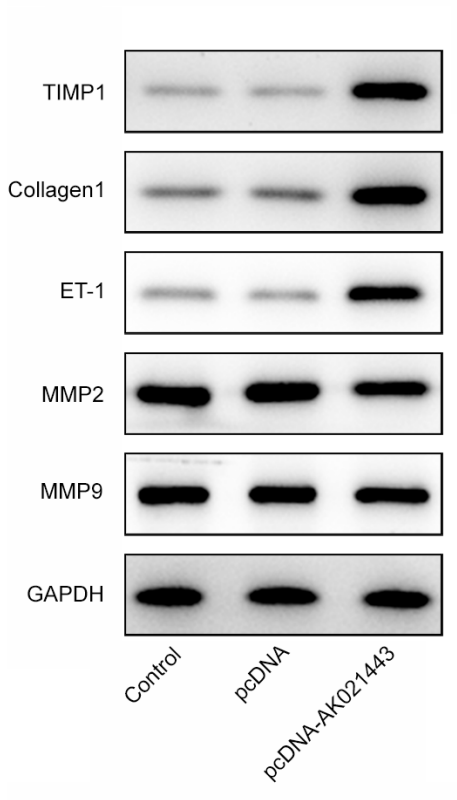

B
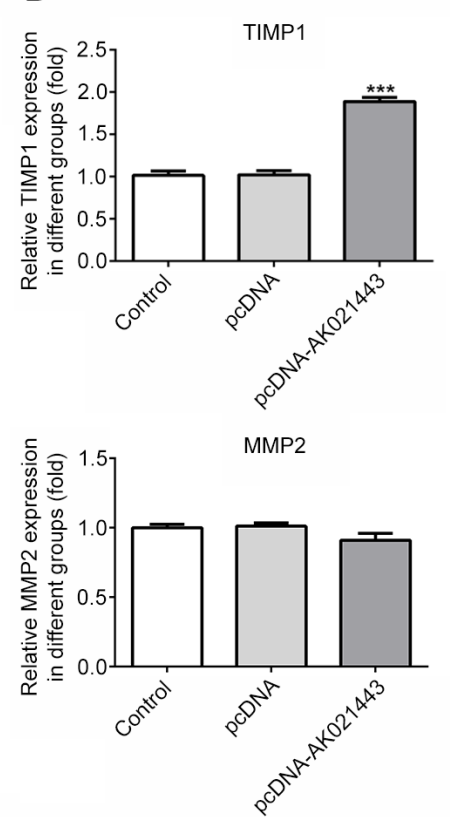
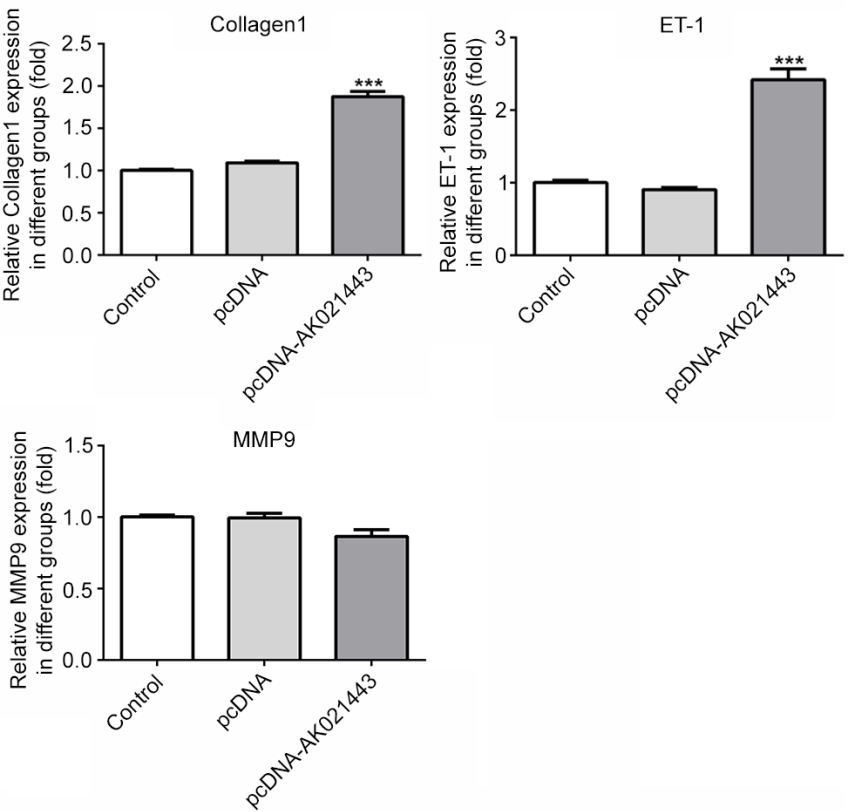

Figure 4. AK021443 overexpression induces extracellular matrix deposition. Protein expression levels were (A) determined by western blotting and (B) semi-quantified for TIMP1, collagen1, ET-1, MMP2 and MMP9. ${ }^{* * * *} \mathrm{P}<0.001 \mathrm{vs.} \mathrm{pcDNA.} \mathrm{TIMP1,} \mathrm{TIMP} \mathrm{metallopeptidase} \mathrm{inhibitor} \mathrm{1;} \mathrm{ET-1,} \mathrm{endothelin} \mathrm{1;}$ MMP, matrix metallopeptidase.

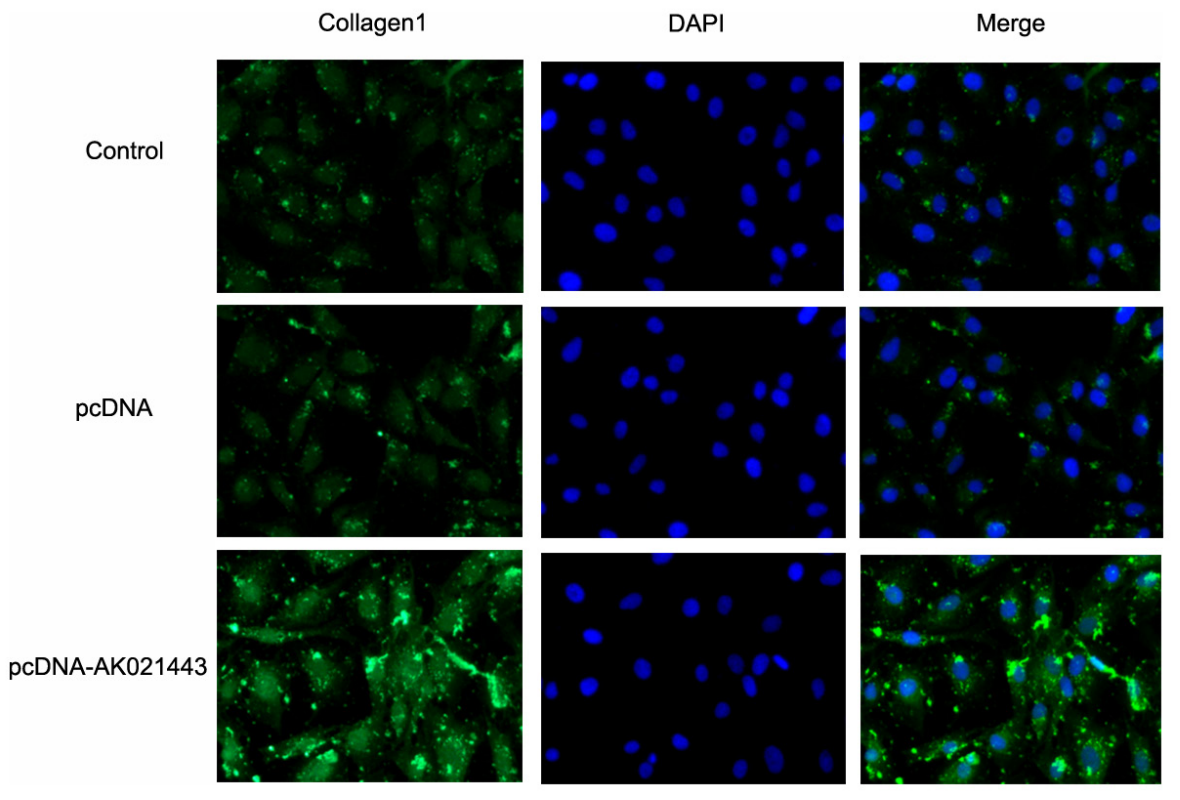

Figure 5. AK021443 overexpression enhances the expression of collagen1. Representative immunofluorescence staining for collagen 1 in the different experimental groups (magnification, $\mathrm{x} 400$ ).

\section{Discussion}

To the best of our knowledge, the present study investigated the association between lncRNA AK021443 and hepatic fibrosis for the first time. The results suggested that AK021443-overexpression LX-2 cells displayed enhanced proliferation, EMT, ECM deposition and inflammatory responses compared with pcDNA-transfected cells, demonstrating the potential role of AK021443 in promoting hepatic fibrosis development.
Extensive studies have revealed that lncRNAs serve a vital role in hepatic fibrosis. For example, lncRNA metastasis associated lung adenocarcinoma transcript 1 promoted HSC activation by blocking the silent information regulator 1 , which mediated the inhibition of the TGF- $\beta$ signaling pathway in the progression of hepatic fibrosis $(20,21)$. In addition, the expression of lncRNA activated by TGF- $\beta$ was increased in the liver tissues and plasma of patients with liver fibrosis, and competed with TGF- $\beta$ receptor II and Smad 2 to bind to microRNA-425-5p to promote collagen formation and HSC activation, thereby 

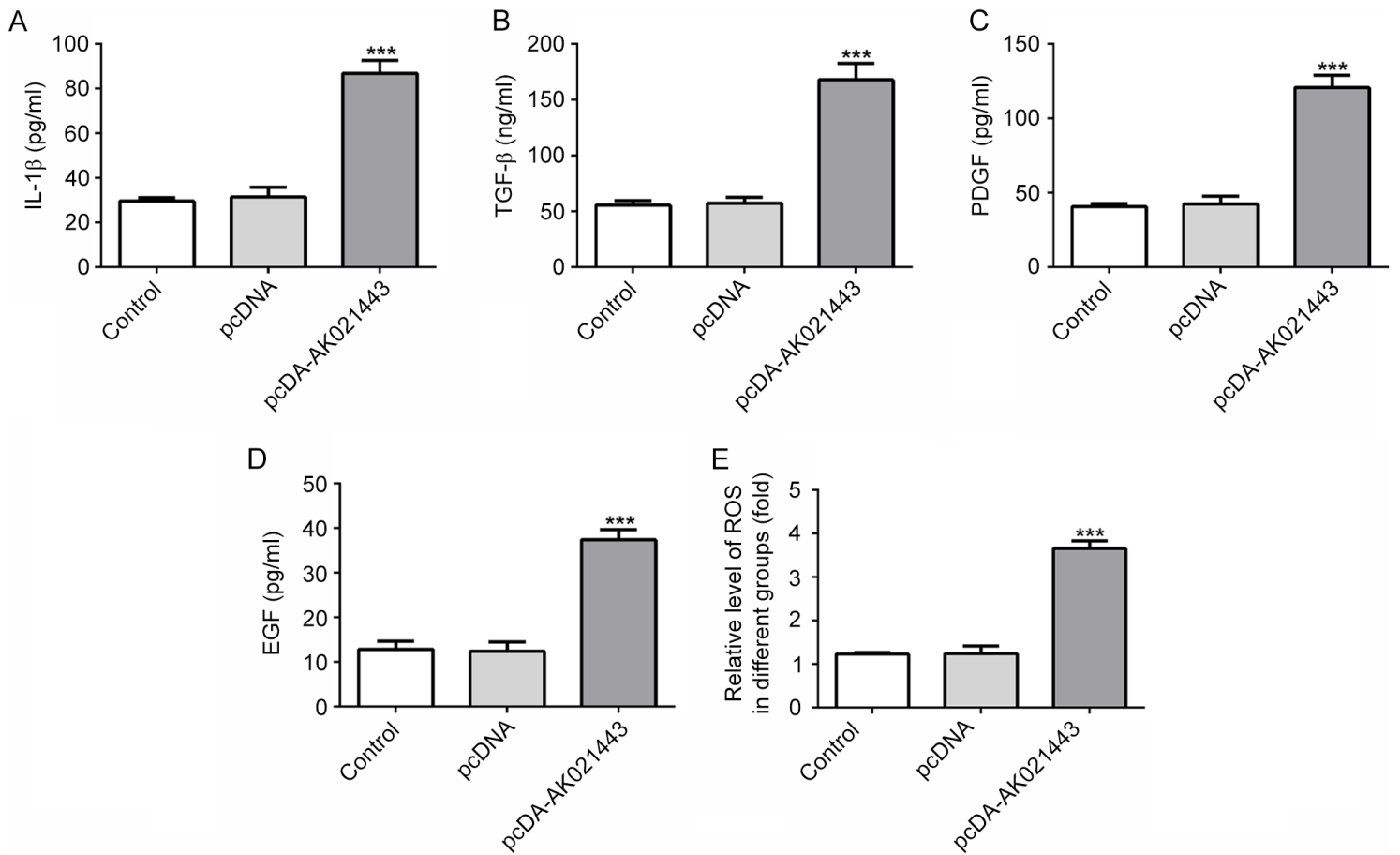

Figure 6. AK021443 overexpression promotes the generation of inflammatory cytokines. Generation of (A) IL-1 $\beta$, (B) TGF- $\beta$, (C) PDGF, (D) EGF and (E) ROS $(n=3) .{ }^{* * *} \mathrm{P}<0.001$ vs. pcDNA. IL-1 $\beta$, interleukin-1 $\beta$; PDGF, platelet derived growth factor; EGF, epidermal growth factor; ROS, reactive oxygen species.

affecting the occurrence of hepatic fibrosis (22). Considering the wide regulatory effects of IncRNAs in hepatic fibrosis and the promoting effect of IncRNA AK021443 in HCC, the present study hypothesized that AK021443 may also serve a potential role in hepatic fibrosis.

Hepatic fibrosis is a chronic pathological process, and the activation of HSCs, the resident perisinusoidal cell type, is typically considered to be a pivotal step in hepatic fibrosis (23). HSCs activated by inflammation-related chemokines were identified as proliferative cells that could transform into fibroblasts (24). Fibroblasts excessively secrete and express collagen, resulting in excessive ECM deposition, thereby contributing to fibrosis (25). Therefore, suppressing HSC activation may serve as a potential therapeutic target for hepatic fibrosis (26). The aim of the present study was to investigate the effects of AK021443 overexpression on HSC activation in the LX-2 cell line.

Firstly, the results indicated that AK021443 overexpression significantly increased cell proliferation, the proportion of $\mathrm{S}$ phase cells and the protein expression levels of CDK2 and cyclin D1, but decreased p21 expression levels compared with the pcDNA group. The cyclin/CDK/CDK inhibitor (CDKI) network serves an important role in the regulation of the $G_{1} / S$ phase during the cell cycle, among which, the cyclin/CDK complex can facilitate the initiation of the cell cycle and promote cell proliferation. By contrast, CDKI p21 can bind to the cyclin/CDK complex, including cyclin $\mathrm{D} / \mathrm{CDK} 4 / 6$ and cyclin E/CDK2, thereby arresting the cell cycle in the $G_{1}$ phase, leading to the inhibition of DNA replication and cell proliferation (27). Therefore, alterations to cell proliferation and cell cycle distribution observed in the present study suggested that AK021443 promoted HSC proliferation.
Subsequently, based on the aforementioned results, which suggested that AK021443 enhanced HSC proliferation, whether AK021443 could induce the transformation of HSC into fibroblasts was investigated. AK021443 overexpression decreased E-cadherin expression and increased the expression of $\mathrm{N}$-cadherin, vimentin and snail compared with the pcDNA group. These proteins are associated with EMT, whereby epithelial cells lose cell adhesion-associated junction proteins, such as E-cadherin and occludin, and gain mesenchymal markers, such as N-cadherin, vimentin and snail, to promote EMT (28). EMT refers to the complex process of epithelial cell transformation into mesenchymal cells. During the process, the polarity of epithelial cells disappears, and invasive and migratory abilities increase, accompanied by the downregulation of epithelial markers and the upregulation of mesenchymal markers (29). On the other hand, the expression levels of TIMP1, collagen 1 and ET-1 were significantly increased, whereas MMP2 and MMP9 expression levels were reduced by AK021443 overexpression compared with the pcDNA group. TIMP, collagen and ET-1 are markers of ECM, the deposition of which serves a profibrotic role in the development of fibrosis (25). MMP2 and MMP9, which can be inhibited by TIMPs, hydrolyze collagen (30). In summary, the results indicated that $\mathrm{AK} 021443$ might function as a promoter of HSC transformation into fibroblasts.

Moreover, the results indicated that AK021443overexpression cells displayed significantly increased release of IL-1 $\beta$, TGF- $\beta$, PDGF, EGF and ROS compared with the pcDNA group. These cytokines participate in the inflammatory response and fibrillogenesis initiated by endogenous and exogenous damage factors during fibrosis, and the release of these cytokines can aggravate inflammation, further promoting fibrosis (7). Moreover, TGF- $\beta$ is a potent stimulator 
of the synthesis of ECM proteins in fibrogenic cells and can stimulate other signaling pathways to regulate fibroblast function (31). The excessive generation of ROS can result in oxidative stress damage, which serves an important role in the development of liver fibrosis (32). In liver fibrosis, HSC activation is induced by the increased generation of oxidative free radicals (33). Therefore, the results indicated that AK021443 induced HSC cell activation and their transformation into myofibroblasts.

In summary, the results of the present study provided a comprehensive understanding of the role of AK021443 in hepatic fibrosis, indicating that AK021443 promoted HSC cell activation, proliferation and transformation into myofibroblast by regulating EMT processes and ECM deposition. The present study provided novel insights into the role of AK021443 in the occurrence and development of hepatic fibrosis in vitro. Collectively, the results indicated that AK021443 upregulation may serve as a promising marker for hepatic fibrosis and AK021443 inhibition may serve as a potential therapeutic approach for hepatic fibrosis.

\section{Acknowledgements}

Not applicable.

\section{Funding}

No funding was received.

\section{Availability of data and materials}

The datasets used and/or analyzed during the current study are available from the corresponding author on reasonable request.

\section{Authors' contributions}

YY and ZM contributed to the conception and design of the study, and acquired and analyzed the data. YY drafted the manuscript and revised it critically for important intellectual content. Both authors read and approved the final manuscript.

\section{Ethics approval and consent to participate}

Not applicable.

\section{Patient consent for publication}

Not applicable.

\section{Competing interests}

The authors declare that they have no competing interests.

\section{References}

1. Lee UE and Friedman SL: Mechanisms of hepatic fibrogenesis. Best Pract Res Clin Gastroenterol 25: 195-206, 2011.

2. Zhou WC, Zhang QB and Qiao L: Pathogenesis of liver cirrhosis. World J Gastroenterol 20: 7312-7324, 2014.
3. Zhang CY, Yuan WG, He P, Lei JH and Wang CX: Liver fibrosis and hepatic stellate cells: Etiology, pathological hallmarks and therapeutic targets. World J Gastroenterol 22: 10512-10522, 2016.

4. Higashi T, Friedman SL and Hoshida Y: Hepatic stellate cells as key target in liver fibrosis. Adv Drug Deliv Rev 121: 27-42, 2017.

5. Tacke F and Weiskirchen R: Update on hepatic stellate cells: Pathogenic role in liver fibrosis and novel isolation techniques. Expert Rev Gastroenterol Hepatol 6: 67-80, 2012.

6. Wang FS, Fan JG, Zhang Z, Gao B and Wang HY: The global burden of liver disease: The major impact of China. Hepatology 60: 2099-2108, 2014.

7. Manka P,Zeller A and Syn WK: Fibrosis in chronic liver disease: An update on diagnostic and treatment modalities. Drugs 79: 903-927, 2019

8. Sui M, Jiang X, Chen J, Yang H and Zhu Y: Magnesium isoglycyrrhizinate ameliorates liver fibrosis and hepatic stellate cell activation by regulating ferroptosis signaling pathway. Biomed Pharmacother 106: 125-133, 2018.

9. Peng H, Wan LY, Liang JJ, Zhang YQ, Ai WB and Wu JF: The roles of lncRNA in hepatic fibrosis. Cell Biosci 8: 63, 2018.

10. Dong Z, Li S, Wang X, Si L, Ma R, Bao L and Bo A: lncRNA GAS5 restrains $\mathrm{CCl}_{4}$-induced hepatic fibrosis by targeting miR-23a through the PTEN/PI3K/Akt signaling pathway. Am J Physiol Gastrointest Liver Physiol 316: G539-G550, 2019.

11. Shen X, Guo H, Xu J and Wang J: Inhibition of lncRNA HULC improves hepatic fibrosis and hepatocyte apoptosis by inhibiting the MAPK signaling pathway in rats with nonalcoholic fatty liver disease. J Cell Physiol 234: 18169-18179, 2019.

12. Xu J, Bai J, Zhang X, Lv Y, Gong Y, Liu L, Zhao H, Yu F, Ping Y, Zhang G, et al: A comprehensive overview of lncRNA annotation resources. Brief Bioinform 18: 236-249, 2017.

13. Yu F, Geng W, Dong P, Huang Z and Zheng J: LncRNA-MEG3 inhibits activation of hepatic stellate cells through SMO protein and miR-212. Cell Death Dis 9: 1014, 2018.

14. Li YC, Wang D and Zhu GY: Increased expression of long noncoding RNA AK021443 predicts worse clinical outcome in hepatocellular carcinoma. Eur Rev Med Pharmacol Sci 22: 4855-4860, 2018.

15. Yang J, Li J, Liu B, Zhang R, Gu F, Zhao J and Cheng S: Long noncoding RNA AK021443 promotes cell proliferation and migration by regulating epithelial-mesenchymal transition in hepatocellular carcinoma cells. DNA Cell Biol 37: 481-490, 2018.

16. Kumar MM and Goyal R: LncRNA as a therapeutic target for angiogenesis. Curr Top Med Chem 17: 1750-1757, 2017.

17. Yang Z, Jiang S, Shang J, Jiang Y, Dai Y, Xu B, Yu Y, Liang Z and Yang Y: LncRNA: Shedding light on mechanisms and opportunities in fibrosis and aging. Ageing Res Rev 52: 17-31, 2019.

18. Livak KJ and Schmittgen TD: Analysis of relative gene expression data using real-time quantitative PCR and the 2(-Delta Delta C(T)) method. Methods 25: 402-408, 2001.

19. Yang H, Shen H, Li J and Guo LW: SIGMAR1/Sigma-1 receptor ablation impairs autophagosome clearance. Autophagy 15: 1539-1557, 2019.

20. Sun L, Fan Z, Chen J, Tian W, Li M, Xu H, Wu X, Shao J, Bian Y, Fang $M$ and $\mathrm{Xu}$ Y: Transcriptional repression of SIRT1 by protein inhibitor of activated STAT 4 (PIAS4) in hepatic stellate cells contributes to liver fibrosis. Sci Rep 6: 28432, 2016.

21. Lee FT, Mountain AJ, Kelly MP, Hall C, Rigopoulos A, Johns TG, Smyth FE, Brechbiel MW, Nice EC, Burgess AW and Scott AM: Enhanced efficacy of radioimmunotherapy with 90Y-CHX-A'-DTPA-hu3S193 by inhibition of epidermal growth factor receptor (EGFR) signaling with EGFR tyrosine kinase inhibitor AG1478. Clin Cancer Res 11: 7080s-7086s, 2005.

22. Fu N, Niu X, Wang Y, Du H, Wang B, Du J, Li Y, Wang R, Zhang Y, Zhao S, et al: Role of LncRNA-activated by transforming growth factor beta in the progression of hepatitis $\mathrm{C}$ virus-related liver fibrosis. Discov Med 22: 29-42, 2016.

23. Trautwein C, Friedman SL, Schuppan D and Pinzani M: Hepatic fibrosis: Concept to treatment. J Hepatol 62 (1 Suppl): S15-S24, 2015.

24. Duval F, Moreno-Cuevas JE, Gonzalez-Garza MT, Rodriguez-Montalvo C and Cruz-Vega DE: Protective mechanisms of medicinal plants targeting hepatic stellate cell activation and extracellular matrix deposition in liver fibrosis. Chin Med 9: 27, 2014.

25. Baglieri J, Brenner DA and Kisseleva T: The role of fibrosis and liver-associated fibroblasts in the pathogenesis of hepatocellular carcinoma. Int J Mol Sci 20: 1723, 2019. 
26. Liu WH, Song FQ, Ren LN, Guo WQ, Wang T, Feng YX Tang LJ and Li K: The multiple functional roles of mesenchymal stem cells in participating in treating liver diseases. J Cell Mol Med 19: 511-520, 2015.

27. Georgakilas AG, Martin OA and Bonner WM: p21: A two-faced genome guardian. Trends Mol Med 23: 310-319, 2017

28. Wang CH, Zhu XD, Ma DN, Sun HC, Gao DM, Zhang N, Qin CD, Zhang YY, Ye BG, Cai H, et al: Flot2 promotes tumor growth and metastasis through modulating cell cycle and inducing epithelial-mesenchymal transition of hepatocellular carcinoma. Am J Cancer Res 7: 1068-1083, 2017.

29. Yamada S, Fuchs BC, Fujii T, Shimoyama Y, Sugimoto H, Nomoto S, Takeda S, Tanabe KK, Kodera Y and Nakao A: Epithelial-to-mesenchymal transition predicts prognosis of pancreatic cancer. Surgery 154: 946-954, 2013.

30. Wynn TA: Common and unique mechanisms regulate fibrosis in various fibroproliferative diseases. J Clin Invest 117: 524-529, 2007.
31. Rockey DC, Bell PD and Hill JA: Fibrosis-a common pathway to organ injury and failure. N Engl J Med 373: 96, 2015.

32. Birben E, Sahiner UM, Sackesen C, Erzurum S and Kalayci O: Oxidative stress and antioxidant defense. World Allergy Organ J 5: 9-19, 2012.

33. Qiu YN, Wang GH, Zhou F, Hao JJ, Tian L, Guan LF, Geng XK, Ding YC, Wu HW and Zhang KZ: PM2.5 induces liver fibrosis via triggering ROS-mediated mitophagy. Ecotoxicol Environ Saf 167: 178-187, 2019.

This work is licensed under a Creative Commons Attribution-NonCommercial-NoDerivatives 4.0 International (CC BY-NC-ND 4.0) License. 